\title{
FUENTES DIGITALES Y FUENTES IMPRESAS. PRÁCTICAS LETRADAS Y PLAGIO EN EL MARCO UNIVERSITARIO
}

\author{
Aurora María Ruiz Bejarano \\ Universidad de Cádiz \\ auroramaria.ruiz@uca.es
}

RESUMEN / ABSTRACT

Los estudios acerca de las prácticas de escritura deshonestas experimentaron un auge significativo con la expansión de las tecnologías de la información y de la comunicación. Una parte de estos estudios acepta la celeridad de las herramientas digitales y la accesibilidad de la información como las principales causas de la comisión del plagio digital. Sin embargo, es necesario realizar una aproximación al ciberplagio que lo sitúe dentro de los márgenes de las transformaciones operadas en las prácticas de escritura y que inciden también en las formas de comprender el conocimiento y de estimar el aprendizaje. Este artículo recoge algunos de los resultados preliminares de una investigación realizada en la Universidad de Cádiz ${ }^{1}$ con el fin de profundizar en la naturaleza de la percepción del plagio y del plagio digital entre el alumnado de los grados de Educación Infantil y Educación Primaria.

PALABRAS CLAVE: ciberplagio, plagio, plagio académico, prácticas de escritura, educación superior.

Studies on dishonest writing practices have experienced a significant growth with the spread of information and communication technologies. Some of these studies accept that the main cause of digital plagiarism is the speed of digital tools and the accessibilityof information. However, it is necessary to carry out an approach to cyber-plagiarism which placeswithin the marginsof the transformations carried out on writing practices and which have an impact on

1 Proyecto Diagnóstico y evaluación del plagio académico en los grados de Educación Infantil y Educación Primaria. Conocer la realidad para mejorarla, dirigido por la profesora Rosa Vázquez Recio y correspondiente a la Convocatoria de Actuaciones Avaladas para la Formación del Profesorado. Convocatoria 2014/2015. 
the way of understanding knowledge and of evaluating learning.This article includes some of the preliminary results of a research carried out in the University ofCádiz with the aim of deepening in the nature of the perception of plagiarism and of digital plagiarism among the students of the Degrees of Infant and Primary Education.

KEYWORDS: cyber-plagiarism; plagiarism; academic plagiarism, writing practices, higher education.

\section{INTRODUCCIÓN}

Los estudios acerca del plagio en el marco universitario y de las prácticas académicas deshonestas experimentaron un auge significativo con el avance de la era digital y con la expansión y consolidación de las tecnologías de la información y de la comunicación (Cerezo 2006; Comas y Sureda 2007, 2008; Ferraz 2008; Sureda, Comas y Morey 2008; Gullifer y Tyson 2010; Hongyan et al. 2007; Sisti 2007; Underwood y Szabo 2003). En ese sentido, la literatura centrada en el estudio del plagio y del ciberplagio subraya la naturaleza impresa o digital de los formatos y de las fuentes como el elemento que diferencia ambos grupos de prácticas deshonestas y que delimita la definición de ambos (Ballano y Muñoz 2014), de modo que:

Adoptar y presentar como propias ideas, teorías e hipótesis de otros no es algo nuevo, pero las tecnologías asociadas a la Sociedad de la Información (SI), sobre todo Internet y más concretamente el World Wide Web (WWW), facilitan enormemente esta práctica éticamente reprobable y académicamente incorrecta (Comasy Sureda 2007).

Los esfuerzos de este corpus de investigaciones se han dirigido hacia cuatro focos elementales de interés.

- Análisis y cuantificación de la prevalencia, caracterización de los alumnos plagiadores y extensión del fenómeno.

- Descripción de los distintos tipos de ciberplagio.

- Análisis de las causas (atribuciones) que lo producen.

- Soluciones y medidas: detección, regulación y prevención (Sureda, Comas y Morey 2008: 199-200).

Sin embargo, en el ámbito más específico de las prácticas letradas, es necesario realizar una aproximación a las prácticas de escritura deshonestas que sitúe 
al plagio y al ciberplagio dentro de los márgenes de las transformaciones contemporáneas que se han operado en las prácticas letradas y que inciden también en las formas de comprender el conocimiento y en el valor que se le otorga al aprendizaje. Este artículo explora de manera preliminar esa cuestión desde dos vertientes complementarias, con el fin de dilucidar las posibilidades de ahondar a posteriori en esa línea de análisis.

En primer lugar, se realiza una reflexión acerca del alcance y de las implicaciones de los cambios producidos en el contexto de las prácticas de lectura y escritura actuales. Este análisis se lleva a cabo contemplando dichos cambios desde la perspectiva más amplia de aquellas otras transformaciones sociales y culturales que tienen especial resonancia en el campo de la educación.

En segundo lugar, el análisis previo no solo tiene valor en sí mismo, sino que pretende, además, servir de marco de referencia donde anclar una investigación realizada en la Universidad de Cádiz y cuyos resultados constituyen la segunda parte de este artículo. Esta investigación tenía como propósito conocer, comprender y profundizar en la naturaleza de la percepción del plagio y del plagio digital entre el alumnado de los grados de Educación Infantil y Educación Primaria. Las reflexiones de este artículo parten de un segmento de los resultados de ese estudio; un segmento compuesto por aquella información que se hallaba relacionada con las prácticas letradas organizadas desde las aulas universitarias y con la comisión del plagio o del ciberplagio académico. Es decir, con aquellas praxis deshonestas donde se produce la copia total o parcial de textos procedentes de fuentes impresas o digitales y que producen en actividades organizadas desde el aula, bien se trate de actividades de clase o bien de actividades para la formación académica fuera del aula. La compra de trabajos académicos, la suplantación durante la celebración de exámenes o la entrega del trabajo de una asignatura cumplida para su valoración en otra asignatura diferente son algunos ejemplos de prácticas académicas deshonestas (Comas et al. 2011) que quedan al margen de este artículo.

\section{2. ¿CAMBIO DE ÉPOCA Y REVOLUCIÓN EN LAS PRÁCTICAS LETRADAS?}

Decía el pensador francés Roger Chartier (2010) que es necesario saber distinguir entre la revolución del libro y las transformaciones que se producen en el campo de la lectura y de sus prácticas sociales, pues se trata, por lo general, 
de procesos no solo diferentes sino también asincrónicos. La invención de la imprenta en el siglo XV, por recurrir a un ejemplo, favoreció la celeridad en la copia de los textos escritos y, en ese sentido, supuso un punto de inflexión importante en la historia del libro. Sin embargo, la aparición del códice no fue simultánea a la creación de la imprenta, pues su origen se remonta a varios siglos atrás, hasta los comienzos de la era cristiana. Del mismo modo, la aparición de la imprenta no alteró sustancialmente las prácticas de lectura, cuya transformación dio comienzo en la Edad Media pero no fue hasta el siglo XVIII cuando se produjo la eclosión de la lectura silenciosa con el avance del individualismo y de la privacidad (Béjar 1995).

La consolidación de la era digital, sin embargo, constituye una transformación peculiar, en la medida en que su naturaleza se ha manifestado muy diferente a la de otras transformaciones de épocas anteriores como la que trajo consigo la invención de la imprenta.

La originalidad de la revolución de nuestro presente reside en que asocia tres transformaciones radicales: propone una nueva técnica de composición, inscripción y comunicación de los textos, impone un nuevo soporte a los textos (la pantalla del ordenador cualesquiera que sean) e impone o sugiere nuevas maneras de leer: discontinuas, fragmentadas, segmentadas. No debemos menospreciar la singularidad de la revolución que nos hace entrar en el mundo digital (Chartier 2010:3).

En efecto, en el campo de la lectura, al igual que en el de la escritura, se han desencadenado intensos cambios que afectan a tres planos diferentes: el de la producción de los textos y su diseminación; el de sus soportes y formatos; y el de las propias formas de afrontar la lectura. Esta serie de transformaciones, sin embargo, no deben contemplarse de manera aislada, como si los acontecimientos que comprometen se ciñesen exclusivamente al ámbito de lo letrado. El espacio público y la ciudadanía se levantan hoy sobre un terreno transido por profundas transformaciones sociales, culturales y económicas con consecuencias especialmente visibles en los ámbitos de la educación formal, no formal e informal. La pluralidad sociocultural de los sujetos educandos devenida principalmente del fuerte empuje de los movimientos migratorios, la pujanza del multiculturalismo y la dilatación de la brecha económica en las ciudades; la aparición de nuevas formas de conocimiento y de acceso a la información, la profunda inmersión de las tecnologías de la comunicación y de la información en las esferas de lo 
cotidiano, la redefinición de los contornos de los espacios públicos y privados, o la aparición de nuevos escenarios de sociabilidad y de ocio son solo algunos de los ejemplos más significativos.

Estas transformaciones actuales han tenido su traducción en los discursos, las simbolizaciones y las prácticas letradas en la escuela y en otros contextos de aprendizaje como la universidad o los entornos digitales. El resultado de estos fuertes procesos transformativos ha sido la creación de un escenario complejo y polifacético donde las pautas canónicas de comprender y practicar la lectura en la escuela conviven con nuevos entornos de aprendizajes, eventos, artefactos y espacios letrados, estrategias de lectura, de escritura y de acceso al conocimiento, formas de sociabilidad y de aprendizaje asociadas a estas praxis emergentes (Martos Núñez y Vivas 2010; Vivas y Martos García 2013; Martos Núñez y Martos García 2014) del mismo modo que, también, han suscitado un profundo debate sobre el plagio, el ciberplagio y las fronteras de la ética.

Asistimos, en definitiva, a una "pluralidad de alfabetismos, de nuevas alfabetizaciones que se encadenan las unas a las otras, de modo que desde la alfabetización básica a la llamada alfabetización informacional, se hace necesaria una visión holística e integradora” (Martos García 2009: 16).

Las organizaciones escolares constituyen, con toda probabilidad, el escenario donde este panorama de contrastes es más acusado. Las tentativas de transformar las bibliotecas escolares en centros de recursos para la enseñanza con la red como fuente de aprendizajes permanentes (Marzal y Cuevas 2007), o la convivencia del libro de texto y la pizarra digital o el ordenador en las aulas son, quizás, los ejemplos más paradigmáticos de esta basculación que se produce entre la cultura impresa (Chartier 2007) y la cibercultura. No obstante, esta convivencia de prácticas letradas de diferente naturaleza es más acusada en la escuela -y también en la universidad- donde, incluso, hay en general una preeminencia del formato impreso; fuera de las aulas, sin embargo, el predominio de lo digital es mayor. Pérez Gómez (2012) ilustra del siguiente modo lo que sucede con las escuelas y que sería igualmente válido para el caso de la universidad, aunque respetando los matices de las diferencias entre ambos espacios:

Cuando los estudiantes contemporáneos abandonan cada día la escuela se introducen en un escenario de aprendizaje organizado de forma radicalmente diferente. En la era global de la información digitalizada el acceso al conocimiento es relativamente fácil, inmediato, 
ubicuo y económico. Uno puede acceder en la red a la información requerida, al debate correspondiente, seguir la línea de indagación que le parezca oportuna $[\ldots]$ y si le apetece puede formar o participar en redes múltiples de personas y colectivos que comparten intereses, informaciones, proyectos y actividades, sin limitaciones de tiempo, institucionales o geográficas. ¿En que [sic] mundo vivimos? (47-48).

\section{METODOLOGÍA DEL ESTUDIO}

La mejora de la escritura académica del alumnado (Romero Oliva 2014) así como la prevención y erradicación del plagio y del ciberplagio constituyen dos pilares elementales de la formación docente en la Universidad de Cádiz. El trabajo sobre las prácticas letradas del estudiantado de los grados de Educación Infantil y Primaria es una constante transversal en su formación académica, puesto que: "los alumnos deben realizar durante sus estudios trabajos de investigación, que por su naturaleza académica exigen un nivel de comunicación alto" y también dado que "el docente tendrá la necesidad de ser modelo en el dominio de las competencias comunicativas (Jiménez, Romero y Sánchez 2014: 26).

En este marco fue aprobado el proyecto Diagnóstico y evaluación del plagio académico en los Grados de Educación Infantil y Educación Primaria. Conocer la realidad para mejorarla, dentro de la Convocatoria de Actuaciones Avaladas para la Formación del Profesorado del curso académico 2014/2015. La investigación de la que ahora se muestran algunos de sus resultados y conclusiones se desarrolló en la Facultad de Ciencias de la Educación de la Universidad de Cádiz. El propósito que orientaba el estudio era el de realizar una aproximación a la percepción del plagio y del plagio digital entre los estudiantes de los grados de Educación Infantil y Educación Primaria. Según recoge la memoria del proyecto, los objetivos específicos de la investigación eran:

1. Identificar el grado de conocimiento que el alumnado universitario tiene del plagio y ciberplagio. Para poder llevar a cabo medidas que mejoren y, por tanto, que reduzcan la práctica del plagio en los estudios universitarios, es imprescindible descubrir qué conocimiento conceptual y práctico tiene el alumnado. 
2. Conocer los motivos que llevan al alumnado a realizar o no esta práctica académica incorrecta. La existencia de esta práctica nos lleva necesariamente a identificar los motivos que conducen al alumnado a realizar la misma. Conocer sus motivos ayudará a tomar medidas que mejoren los procesos de enseñanza y aprendizaje.

3. Determinar los factores que pueden incidir en que el alumnado lleve a cabo esta práctica. El plagio académico se presenta como un fenómeno complejo en tanto que no es una práctica que responda solo a la intencionalidad del alumnado, sino que también pueden intervenir otros factores (sociales, género, culturales, contextuales).

Los cuestionarios se aplicaron en los cuatro cursos de ambas titulaciones a una muestra de 539 sujetos $(\mathrm{n}=539)$ sobre una población total de 745 estudiantes (Tabla 1). En la muestra en la que fue aplicado el cuestionario se aprecia un porcentaje mucho mayor de mujeres $(83,9 \%)$ que de hombres $(16,1 \%)$ estudiando el grado de Infantil y el grado de Primaria. La edad media de los estudiantes es de 22 años. No se aprecia una diferencia a considerar entre el número de estudiantes de cada uno de los grados participantes en el proyecto: un 56,3\% pertenecían al grado de Educación Infantil y un 43,7\% al de Educación Primaria).

Tabla 1. Estudiantes participantes en la investigación

\begin{tabular}{|llrrrr|}
\hline & & Frecuencia & Porcentaje & $\begin{array}{c}\text { Porcentaje } \\
\text { válido }\end{array}$ & $\begin{array}{r}\text { Porcentaje } \\
\text { acumulado }\end{array}$ \\
\hline Válido & M. Educ. Infantil & 301 & 55,8 & 56,3 & 56,3 \\
& M. Educ. Primaria & 234 & 43,4 & 43,7 & 100,0 \\
& Total & 535 & 99,3 & 100,0 & \\
Perdidos & Sistema & 4 &, 7 & & \\
Total & & 539 & 100,0 & & \\
\hline
\end{tabular}

Los datos fueron explotados con el programa estadístico IBM SPSS Statistics, versión 19.0. 


\section{PRÁCTICAS LETRADAS, PLAGIO Y CIBERPLAGIO ENTRE EL ALUMNADO UNIVERSITARIO}

\subsection{ESCRITURA Y DOCENCIA UNIVERSITARIA: UNA PERSPECTIVA GLOBAL}

La escritura tiene un peso importante entre las actividades que se organizan desde las aulas universitarias. En la Tabla 2 están recogidas aquellas prácticas letradas académicas que se consideraron en el cuestionario. Es preciso contemplar el abanico general de estas prácticas letradas a fin de comprender el panorama global en el que se inserta la comisión del plagio y del plagio digital.

Tabla 2. Prácticas letradas en aulas universitarias

\begin{tabular}{|l|}
\hline \multicolumn{1}{|c|}{ Actividades de clase } \\
\hline Realizar actividades del libro de texto o de otros materiales escritos \\
\hline Realizar exámenes \\
\hline Realizar trabajos de investigación \\
\hline Elaborar presentaciones y realizar exposiciones \\
\hline Buscar y consultar fuentes impresas u on-line y tomar notas sobre su contenido \\
\hline Realizar comentarios de textos \\
\hline Realizar composiciones o redacciones abiertas o creativas \\
\hline Indagar o investigar on-line (explorando páginas web, blogs) \\
\hline
\end{tabular}

Al respecto, un 40,9\% de los estudiantes considera bastante frecuente la realización de actividades procedentes de libros de texto y de otros materiales escritos y un $26,5 \%$ sostiene que esta actividad es muy frecuente en la universidad.

Por su parte, un 54,4\% de los sujetos encuestados considera muy frecuente la realización de exámenes y un 21,1\% mantiene que se trata de una actividad bastante frecuente. Aunque, ciertamente, en la pregunta no aparecía explícita la naturaleza oral o escrita de la prueba, debe tenerse en cuenta que el segundo tipo de pruebas está muy extendido en la educación superior. 
La elaboración de presentaciones para su posterior exposición en clase es otra de las actividades predominantes en las aulas universitarias. Un 42,5\% del alumnado indica que es una práctica bastante frecuente, mientras que un $36,3 \%$ estima que se trata de una actividad muy frecuente.

En lo que concierne a la búsqueda y consulta de información en fuentes impresas o digitales, un 37,7\% de los sujetos encuestados valora esta actividad como bastante frecuente y, en esa misma línea, un 35,3\% sostiene que es muy frecuente. No obstante, cabe destacar que un $22,3 \%$ considera que esta es una actividad poco frecuente (Tabla 3 ). La misma circunstancia se da con los datos referentes a la indagación o investigación digital a través de páginas webs o blogs. Un $34,4 \%$ de los estudiantes apunta que es una actividad bastante frecuente y un $32,7 \%$ afirma que es muy frecuente. Un $24,8 \%$ del estudiantado, por su parte, valora esta práctica como poco frecuente (Tabla 4). A la luz de estos datos se puede entender que la exploración, la búsqueda y la consulta de información de fuentes digitales e impresas constituyen actividades relevantes en el ámbito universitario. En ambos tipos de actividades se debe considerar que, a diferencia de lo que sucede con otras prácticas, la búsqueda de información en fuentes impresas o digitales y la investigación en entornos digitales dependen en mayor medida de la iniciativa del alumnado, lo que podría ayudar a comprender el porcentaje de estudiantes que considera poco frecuentes estas actividades en el marco universitario (Tabla 4).

\section{Durante la carrera universitaria: "Buscar y consultar fuentes impresas u on_line y tomar notas sobre su contenido".}

Tabla 3. Consulta de fuentes impresas y digitales

\begin{tabular}{|llrrrr|}
\hline & & Frecuencia & Porcentaje & $\begin{array}{c}\text { Porcentaje } \\
\text { válido }\end{array}$ & $\begin{array}{c}\text { Porcentaje } \\
\text { acumulado }\end{array}$ \\
\hline \multirow{4}{*}{ Válido } & Muy poco frecuente & 25 & 4.6 & 4.6 & 4.6 \\
& Poco frecuente & 120 & 22.3 & 22.3 & 27.0 \\
& Bastante frecuente & 203 & 37.7 & 37.7 & 64.7 \\
& Muy frecuente & 190 & 35.3 & 35.3 & 100.0 \\
& Total & 538 & 99.8 & 100.0 & \\
Perdidos & Sistema & 1 & .2 & & \\
Total & & 539 & 100.0 & & \\
\hline
\end{tabular}




\section{Durante la carrera universitaria: "Indagar o investigar on_line (explorando páginas web, blogs)".}

Tabla 4. Investigar en la Red

\begin{tabular}{|ll|r|r|r|r|}
\hline & Frecuencia & Porcentaje & $\begin{array}{c}\text { Porcentaje } \\
\text { válido }\end{array}$ & $\begin{array}{c}\text { Porcentaje } \\
\text { acumulado }\end{array}$ \\
\hline \multirow{4}{*}{ Válido } & Muy poco frecuente & 43 & 8.0 & 8.1 & 8.1 \\
& Poco frecuente & 131 & 24.3 & 24.8 & 32.9 \\
& Bastante frecuente & 182 & 33.8 & 34.4 & 67.3 \\
Total & Muy frecuente & 173 & 32.1 & 32.7 & 100.0 \\
Perdidos & Sistema & 529 & 98.1 & 100.0 & \\
Total & & 10 & 1.9 & & \\
\hline
\end{tabular}

La redacción de comentarios de texto, por su parte, es valorada poco frecuente por un $40,6 \%$ del alumnado y muy poco frecuente por un $25,8 \%$, lo que puede explicarse atendiendo a la posibilidad de que se trate de una actividad residual desarrollada en asignaturas muy concretas. La realización de ensayos, composiciones libres o redacciones creativas es otra de las actividades con una baja frecuencia. Un 46,9\% de los sujetos encuestados mantiene que se trata de una actividad poco frecuente y un $25,5 \%$ la considera muy poco frecuente.

En lo referente a la preparación y ejecución de trabajos de investigación, un $33,6 \%$ de estudiantes la considera una práctica poco frecuente en la universidad, mientras que un $33,2 \%$ estima que se trata de una actividad bastante frecuente. Al mismo tiempo, un 14,5\%mantiene que la elaboración de trabajos de investigación es muy poco frecuente, frente al 18,5\% que la considera muy frecuente. Una explicación puede hallarse en el hecho de que el alumnado desconoce con exactitud qué es y cómo se realiza este tipo de trabajos. El 40,4 \% de los encuestados sostiene que nunca se le ha enseñado preparar trabajos académicos, ni en la universidad ni en estudios anteriores. Por su parte, un 43,2\% afirma que ha aprendido a realizar trabajos de esta índole en el contexto de la universidad (Tabla 5). De hecho, como se verá en el próximo apartado, este es uno de los argumentos que esgrimen los estudiantes para justificar la comisión del plagio: el desconocimiento de las pautas y normas que rigen la preparación de un trabajo académico. 


\section{¿Consideras que, ya sea en el instituto o en la universidad, te han enseñado o explicado, de forma adecuada a realizar trabajos académicos?}

Tabla 5. Aprendizaje previo en trabajos académicos

\begin{tabular}{|llrrrr|}
\hline & & Frecuencia & Porcentaje & $\begin{array}{r}\text { Porcentaje } \\
\text { válido }\end{array}$ & $\begin{array}{c}\text { Porcentaje } \\
\text { acumulado }\end{array}$ \\
\hline \multirow{5}{*}{ Válido } & Sí- Secundaria & 27 & 5.0 & 5.1 & 5.1 \\
& Sí- Universidad & 230 & 42.7 & 43.2 & 48.3 \\
& Sí- Secundaria y & 59 & 10.9 & 11.1 & 59.4 \\
& Universidad & & & & \\
& No & 215 & 39.9 & 40.4 & 99.8 \\
& 5 & 1 & .2 & .2 & 100.0 \\
& Total & 532 & 98.7 & 100.0 & \\
Perdidos & Sistema & 7 & 1.3 & & \\
Total & & 539 & 100.0 & & \\
\hline
\end{tabular}

4.2. Prácticas letradas, plagio y ciberplagio: razones en el contexto UNIVERSITARIO

Es cierto que la fácil disponibilidad de la información en la red es una de las razones que los estudiantes universitarios plantean como causa de la comisión del plagio. Un 29,9\% considera este hecho relevante y un $26,6 \%$ bastante relevante. Del mismo modo, la saturación y la falta de tiempo para efectuar los trabajos se presenta como otra de las causas. Un 33,8\% de los estudiantes sostiene que este es un hecho bastante relevante y un $32,9 \%$, muy relevante. Sin embargo, interesa especialmente explorar aquellos factores que guardan mayor relación con las prácticas letradas del alumnado y que explican la recurrencia del plagio y del ciberplagio.

En el análisis de las causas del plagio y del ciberplagio académico, así como en la valoración de su percepción entre el estudiantado en relación con las prácticas letradas y con las formas en que el conocimiento y el aprendizaje son concebidos se perciben dos líneas interesantes de explorar.

En primer lugar se halla el grado de información -o de desconocimientoque el alumnado tenga acerca de las normas existentes para citar correctamente las fuentes y para referenciar las obras consultadas. En segundo lugar está el 
grado de conocimiento que tengan el estudiantado sobre el tipo de trabajo que se le solicita, así como la valía y estima en que lo consideren.

En ese sentido, uno de los primeros ámbitos a explorar es el conocimiento que tienen los estudiantes sobre el citado de las fuentes y la recogida de las referencias bibliográficas. Un $88,7 \%$ de los estudiantes afirma que se les ha enseñado a recoger referencias bibliográficas; el 6,1\% atribuye esta labor al profesorado y el $4 \%$ al personal de biblioteca. Es decir, existe un porcentaje muy alto de población estudiantil que conoce la importancia de recoger convenientemente las fuentes bibliográficas. Sin embargo, solo un $51,1 \%$ del alumnado afirma que sabe reconocer las citas correctas, a lo que se debe añadir que algo menos de la mitad de los sujetos encuestados (un 46,8\%) mantiene que conoce, concretamente, la normativa de la American Psychological Association (APA). En esta misma dirección se puede incluir un dato más: el $31,0 \%$ de los estudiantes considera muy poco relevante el hecho de que internet sea público como uno de las motivaciones del plagio y un $28,3 \%$ lo considera poco relevante (Tabla 6). Es decir, pese a que el alumnado parece reconocer que la autoría de las obras de fuentes digitales es tan legítima como la proveniente de fuentes impresas y pese a que ha recibido formación sobre las normas que rigen el citado de las fuentes, solo la mitad del alumnado sabe citar las fuentes que utiliza en sus trabajos académicos y en sus actividades de aula.

\section{A la hora de realizar trabajos académicos, causas por las que se copia: "Porque existe la creencia de que se puede copiar algo de Internet porque se considera público".}

Tabla 6. Carácter público de internet y plagio

\begin{tabular}{|llrrrr|}
\hline & & Frecuencia & Porcentaje & $\begin{array}{c}\text { Porcentaje } \\
\text { válido }\end{array}$ & $\begin{array}{c}\text { Porcentaje } \\
\text { acumulado }\end{array}$ \\
\hline \multirow{4}{*}{ Válido } & Muy poco relevante & 167 & 31.0 & 31.1 & 31.1 \\
& Poco relevante & 152 & 28.2 & 28.3 & 59.4 \\
& Relevante & 134 & 24.9 & 25.0 & 84.4 \\
& Bastante relevante & 50 & 9.3 & 9.3 & 93.7 \\
& Muy relevante & 34 & 6.3 & 6.3 & 100.0 \\
Perdidos & Total & 537 & 99.6 & 100.0 & \\
Total & Sistema & 2 & .4 & & \\
\hline
\end{tabular}


En lo que respecta a las cuestiones planteadas acerca de las prácticas letradas del aula y la comisión del plagio y del plagio digital, hay algunos datos que resultan importantes subrayar.

En primer lugar, un 31,0\% de los encuestados considera relevante el hecho de no saber cómo se realizan trabajos académicos y un $25,9 \%$, bastante relevante. Solo un $7,7 \%$ lo considera muy poco relevante y un $16,5 \%$, poco relevante (Tabla 7).

\section{A la hora de realizar trabajos académicos, EIMP.Causas por las que se copia: "Porque no se sabe muy bien cómo realizar trabajos académicos".}

Tabla 7. Trabajos académicos y plagio

\begin{tabular}{|llrrrr|}
\hline & & Frecuencia & Porcentaje & $\begin{array}{c}\text { Porcentaje } \\
\text { válido }\end{array}$ & $\begin{array}{c}\text { Porcentaje } \\
\text { acumulado }\end{array}$ \\
\hline \multirow{6}{*}{ Válido } & Muy poco relevante & 41 & 7.6 & 7.7 & 7.7 \\
& Poco relevante & 88 & 16.3 & 16.5 & 24.2 \\
& Relevante & 165 & 30.6 & 31.0 & 55.2 \\
& Bastante relevante & 138 & 25.6 & 25.9 & 81.1 \\
& Muy relevante & 101 & 18.7 & 18.9 & 100.0 \\
Perdidos & Total & 533 & 98.9 & 100.0 & \\
Total & Sistema & 6 & 1.1 & & \\
\hline
\end{tabular}

En segundo lugar, un 30,8\% considera que la naturaleza teórica del trabajo es un factor relevante en la justificación del plagio y del ciberplagio. Un 24,4\%, por su parte, considera el hecho bastante relevante. En esa misma línea, un $28,4 \%$ estima como motivo bastante relevante del plagio o del plagio digital la circunstancia de que se planteen trabajos de recopilación de información, en lugar de trabajos de elaboración propia o creativos; un tipo de trabajo que, de por sí, tiene una incidencia muy baja en las aulas universitarias, según se apreció anteriormente. Un porcentaje muy similar al anterior (el 28,6\%) considera que la naturaleza teórica del trabajo es un hecho relevante en la explicación del plagio y del plagio digital (Tabla 8). 


\section{A la hora de realizar trabajos académicos, EIMP. Causas por las que se copia: "Por el hecho de que el trabajo que se pide sea, básicamente, de tipo teórico".}

Tabla 8. Trabajos teóricos y plagio

\begin{tabular}{|llllll|}
\hline & & Frecuencia & Porcentaje & $\begin{array}{c}\text { Porcentaje } \\
\text { válido }\end{array}$ & $\begin{array}{l}\text { Porcentaje } \\
\text { acumulado }\end{array}$ \\
\hline \multirow{6}{*}{ Válido } & Muy poco relevante & 43 & 8.0 & 8.1 & 8.1 \\
& Poco relevante & 119 & 22.1 & 22.3 & 30.4 \\
& Relevante & 164 & 30.4 & 30.8 & 61.2 \\
& Bastante relevante & 130 & 24.1 & 24.4 & 85.6 \\
& Muy relevante & 77 & 14.3 & 14.4 & 100.0 \\
Perdidos & Total & 533 & 98.9 & 100.0 & \\
Total & Sistema & 6 & 1.1 & & \\
\hline
\end{tabular}

\section{PARA FINALIZAR}

Hay una serie de ideas desarrolladas en este trabajo que es interesante retomar ahora a modo de síntesis, a fin de lograr una visión de conjunto.

La consolidación de la era digital ha llevado consigo la emergencia de nuevos artefactos, eventos y espacios letrados que no han desplazado a los ya existentes. Ha generado, del mismo modo, nuevas formas de sociabilidad y reorientado el debate sobre el alcance de la ética en las prácticas letradas. La complejidad de este escenario es más acuciada en entornos como la escuela y la universidad, que se desplazan entre la cultura impresa y la cultura digital.

La convivencia de prácticas letradas que arraigan en la cultura impresa y en la cultura digital se refleja, sin ir más lejos, en las aulas universitarias, donde los exámenes, la composición de trabajos académicos o la preparación de exposiciones comparten espacio con la investigación en la red o la búsqueda de información en fuentes digitales e impresas.

Precisamente, algunas de estas prácticas letradas son valoradas por el alumnado universitario como factores influyentes en la comisión del plagio y del ciberplagio. Es especialmente relevante el hecho de que los trabajos académicos, especialmente los de naturaleza teórica (un 31\% lo consideran 
relevante) y que requieren una búsqueda intensiva de información (considerado bastante relevante por un 28,4\%) -en fuentes digitales o impresas-favorecen en mayor medida la comisión del plagio. Se da la circunstancia, además, de que pese a que un $88,7 \%$ dice haber recibido instrucción sobre cómo se cita y cómo se recogen las referencias bibliográficas, solo el 51,1\% dice saber reconocer de forma correcta las citas. Y ambas acciones -citar y recopilar las referencias bibliográficas-son, precisamente, muy necesarias para desarrollar convenientemente ese tipo de trabajos académicos.

Enlazando con la primera parte del artículo, el incremento del plagio en los trabajos académicos y teóricos que precisan de una profusa búsqueda de información nos debe hacer reflexionar si la cuestión que hay tras ello es el hecho de que este tipo de trabajos académicos requiere de unas formas de lectura muy diferentes a las predominantes en la era digital y que son, en definitiva, las prácticas en las que están profundamente inmersos los estudiantes fuera de las escuelas, como apuntaba Pérez Gómez (2012) y de las aulas universitarias. Es decir, la recurrencia al plagio en los trabajos académicos plantea sobre la mesa la cuestión de si el problema estriba en que las formas de lectura que entran en juego en este tipo de prácticas universitarias están muy alejadas de esas otras formar de leer vibrantemente contemporáneas que llamaba Chartier (2010) “discontinuas, fragmentadas, segmentadas" (3).

\section{BIBLIOGRAFÍA}

Ballano Olano, Inmaculada e Itziar Muñoz Aldama. "Competencia lecto-escritora y plagio: los desafíos de la era digital". El plagio académico en Educación Secundaria: características del fenómeno y estrategias de intervención. Coord. Comas, Rubén y Jaume Sureda. Islas Baleares: Grupo de Investigación dela Universidad de las Islas Baleares "Educación y Ciudadanía" (2014): 8-25.

Béjar, Helena. El ámbito íntimo. Privacidad, individualismo y modernidad. Madrid: Alianza Editorial, 1995.

Cerezo, Héctor. "Aspectos éticos del plagio académico de los estudiantes". Elementos: Ciencia y Cultura 13/61 (2006): 31-35.

Chartier, Roger. “¿La muerte del libro? Orden del discurso y orden de los libros”. Co-herencia 4 (2007): 119-129. view/14/10

"Entrevista". Álabe 1-Junio (2010). http://revistaalabe.com/index/alabe/article/

Comas, Rubén y Jaume Sureda. "Ciber-plagio académico. Una aproximación al estado de los conocimientos". Revista Textos de la CiberSociedad 10 (2007) http://www.cibersociedad. net/textos/articulo.php?art=121 
"Academic Cyberplagiarism. Tracing the causes to Reach Solutions". Digithum, The Humanities in the Digital Era 10 (2008) http://www.uoc.edu/digithum/10/dt/eng/ comas_sureda.pdf

Comas, Rubén et al. "La integridad académica entre el alumnado universitario español". Estudios Pedagógicos XXXVII 1 (2011): 207-225.

Ferraz, Obdália. "Entre el plagio y la autoría: ¿cuál es el papel de la universidad?”. Revista Brasileira de Educação 13/38 (2008): 357-414.

Gullifer, Judith y Tyson, Graham. A. "Exploring university students' perceptions of plagiarism: a focus group study". Studies in Higher Education 35/4 (2010): 463-481.

Hongyan, Maet al. "An Empirical Investigation of Digital Cheating and Plagiarism among Middle School Students”. American Secondary Education 35/2 (2007): 69-89.

Jiménez Fernández, Rafael; Manuel Fco. Romero Oliva y Susana Rodríguez Sánchez. "Diagnóstico y evaluación de la comunicación escrita en el perfil de ingreso de los grados en Educación Infantil y en Educación Primaria”. La escritura académica: diagnóstico y propuestas de actuación. Una visión desde los grados de magisterio. Coord. Manuel F. Ramos Oliva. Barcelona: Octaedro, (2014): 25-62.

Martos García, Alberto. "Tecnologías de la palabra en la era digital: de la cultura letrada a la cibercultura". Revista Latinoamericana de Tecnología Educativa-RELATEC 8 /2 (2009): 15-37.

"Los jóvenes ante las pantallas: nuevos contenidos y nuevos lenguajes para la educación literaria”. Revista Qurriculum 24 (2011): 11-28.

Martos Núñez, Eloy y Alberto Martos García. “Artefactos culturales y alfabetización en la era digital: discusiones conceptuales y praxis educativa". Teoría de la educación. Revista Interuniversitaria 26 (2014): 119-135.

Martos Núñez, Eloy y Agustín Vivas. Performance, lectura y escritura (Conceptos y prácticas). Coords. Madrid: Universidad Complutense, Red Internacional de Universidades Lectoras, Colección Temas de Lectura, 2010.

Marzal, Miguel y Aurora Cuevas. "Biblioteca escolar para la sociedad del conocimiento en España”. Ci. Inf., Brasília 36 (2007): 54-68.

Pérez Gómez, Ángel I. Educarse en la era digital. Madrid: Morata, 2012.

Romero Oliva, Manuel Fco. La escritura académica: diagnóstico y propuestas de actuación. Una visión desde los grados de magisterio. Coord. Barcelona: Octaedro, 2014.

Sisti, Dominic. A. "How Do High School Students Justify Internet Plagiarism?”. Ethics \& Behavior 17/3 (2007): 215-231.

Sureda, Jaume; Ruben Comas y Mercè Morey. "Cyberplagiarism Webbliography. References to Academic Cyberplagiarism on the Internet". Digithum, The Humanities in the Digital Era 10 (2008) http://www.uoc.edu/digithum/10/dt/eng/comas_sureda.pdf

Underwood, Jean y Attila Szabo. “Academic offences and e-learning: individual propensities in cheating". British Journal of Educational Technology 34/4 (2003): 467-477.

Vivas, Agustín y Aitana Martos García. "Cultura Letrada y Cibercultura". Diccionario de nuevas formas de lectura y escritura. Coord. Eloy Martos Núñez y Mar Campos. Madrid: Red Internacional de Universidades Lectoras-Santillana, (2013): 160-164. 